\title{
Collagen sponge prolongs taurine release for improved wound healing through inflammation inhibition and proliferation stimulation
}

\author{
Liang Wu ${ }^{1,2 \#}$, Qianwen Zhang ${ }^{2 \#}$, Yuan $\mathrm{Li}^{3}$, Wenxiang Song ${ }^{4}$, Anqi Chen ${ }^{2,5}$, Jingjing Liu ${ }^{2}$, Xuan Xuan ${ }^{2}$ \\ ${ }^{1}$ Anqing Municipal Hospital, Anqing, China; ${ }^{2}$ Department of Dermatology, The First Affiliated Hospital of Wenzhou Medical University, Wenzhou, \\ China; ${ }^{3}$ Department of Burn, The First Affiliated Hospital of Wenzhou Medical University, Wenzhou, China; ${ }^{4}$ College of Chemistry and Materials \\ Engineering, Wenzhou University, Wenzhou, China; ${ }^{5}$ School of Pharmaceutical Sciences, Key Laboratory of Biotechnology and Pharmaceutical \\ Engineering, Wenzhou Medical University, Wenzhou, China \\ Contributions: (I) Conception and design: X Xuan, L Wu, J Liu; (II) Administrative support: X Xuan; (III) Provision of study materials or patients: \\ X Xuan, L Wu; (IV) Collection and assembly of data: L Wu, Q Zhang; (V) Data analysis and interpretation: Q Zhang, Y Li, W Song, A Chen; (VI) \\ Manuscript writing: All authors; (VII) Final approval of manuscript: All authors. \\ \#These authors contributed equally to this work. \\ Correspondence to: Jinging Liu; Xuan Xuan. Department of Dermatology, The First Affiliated Hospital of Wenzhou Medical University, Wenzhou, \\ China. Email: liujingjing312@126.com; XuanXuanWY@hotmail.com.
}

\begin{abstract}
Background: Attenuating oxidative stress response is an effective strategy for the treatment of wounds. Taurine is a widely abundant amino acid in mammal species, capable of inhibiting oxygen-free radicals during the inflammation phase.

Methods: A novel taurine carried biocompatible composite collagen-derived sponge, Tau@Col, was fabricated for the treatment of a full-thickness removal mouse wounds model. In vitro experiments included taurine release from Tau@Col and cell viability when co-cultured with Tau@Col. With the prolonged release of taurine upon the wound site, Tau@Col was engineered to perform well in the wound site through inflammation inhibition and proliferation stimulation as demonstrated by a series of histological staining.

Results: In vitro taurine release profile and good cell biocompatibility of Tau@Col were demonstrated. In vivo studies showed that Tau@Col indeed sped up the process of wound regeneration through enhanced granulation formation, collagen deposition as well as re-epithelialization. Further investigations through immunofluorescence staining revealed that the improved wound healing ability of Tau@Col was mediated by the enhanced cell proliferation via the upregulation of endogenous vascular endothelial growth factor (VEGF) and transforming growth factor beta (TGF- $\beta$ ) expression as well as decreased inflammatory response through stimulated M2 polarization of macrophages.
\end{abstract}

Conclusions: This engineered Tau@Col delivery system has great potential as a wound dressing in future applications.

Keywords: Collage sponge; wound healing; taurine; antioxidative; macrophage polarization

Submitted Apr 29, 2021. Accepted for publication Jun 15, 2021.

doi: $10.21037 /$ atm-21-2739

View this article at: https://dx.doi.org/10.21037/atm-21-2739

\section{Introduction}

The skin is the largest organ of the human body. The typical wound healing process can be conveniently divided into 3 overlapping and continuous phases: inflammation, proliferation, and tissue remodeling. In the inflammatory phase particularly, neutrophils and macrophages could generate reactive oxygen species (ROS) for killing pathogens (1), However, excessive production of ROS 
may impair the physiological wound healing process by abundant neutrophil infiltration caused by free radicals (2). The imbalance between ROS and its detoxification process will occur in the ensuing proliferation stage, resulting in excessive oxidative stress within the wound site (3). This often leads to inactivation of free radical scavenger enzymes, cell apoptosis or necrosis with severe lipid peroxidation, as well as mitochondrial and DNA damage upon wound site $(1,4,5)$. Thus, attenuating oxidative stress response has great potential for both acute and chronic wounds treatment. Thus far, many antioxidants, such as vitamins, folic acid, lipoic acid, and glutathione, along with traditional Chinese medicines (TCM), like Ginkgo biloba, Salvia miltiorrhiza, and Panax notoginseng have been used to eliminate ROS on wound healing $(6,7)$. Furthermore, a variety of wound dressings based on synthetic biomaterials have been developed with antioxidant properties for improving healing outcome (8-10). The development of wound dressing with antioxidative property shows a very promising future in clinical applications $(11,12)$.

Due to its unique the antioxidative and anti-inflammatory properties, taurine (Tau), a naturally occurring amino acid, has been widely valued for its anti-inflammatory effects. Taurine is a sulfur-containing amino acid, which exists in abundance in almost all tissues in mammal species $(13,14)$ and is capable of suppressing ROS and oxygen free radical levels during the inflammation stage $(15,16)$. As a direct antioxidant, taurine can significantly reduce lipid peroxidation, and as an indirect antioxidant, it can stabilize the plasma membrane. It is widely acknowledged that taurine plays a role in the innate anti-inflammatory effect, as a high concentration of taurine can found in inflammatory lesions (17). In the immune system, taurine plays a major role due to its enduring anti-oxidative effect (18-20). Taurine has also been used to prevent oxidant damage in many tissues, including incisional skin wounds (21), while topical formulations containing taurine have already been shown to enhance wound healing of the skin tissue (13), gingiva (2), maxillary mucosa (22), as well as periodontal tissue (23). According to these reports, Tau has positive impacts on the inflammatory skin lesions and can help enhancing fibroblast proliferation, collagenous fiber bundle synthesis, and re-angiogenesis in the dermal wounds. Moreover, it is suggested that taurine also can help improve the rate of reepithelialization through a calcium-dependent mechanism upon wound healing $(2,24)$.

The ideal delivery system for taurine should be able to maintain the stability of taurine and slowly release taurine to reduce the frequency of administration (25). Thus, in this study, taurine was loaded into rat tail tendon-derived collagen sponges (Col) to form a Tau@Col dressing. Collagen serves as a predominant component of the ECM of the skin tissue, which better mimics the natural skin environment. Moreover, a dry sponge dressing could absorb more exudate from the wound as well as maintain a moist environment for skin repair. This Tau@Col sponge contained both antioxidative and anti-inflammatory properties from the addition of taurine. This Tau@Col was then applied to a full-thickness wound model of mice in vivo. Data from experiments indicated that the Tau@ Col group exhibited fastest wound closure, along with the best granulation formation and collagen deposition. The improved wound healing property was further demonstrated by its enhanced re-epithelialization ability associated with relevant cell proliferation rate, upregulated endogenous growth factor expressions, and increased polarization of M2 macrophages. Overall, the Tau@Col scaffold had an excellent therapeutic effect on dermal wounds and shows great potential for clinical use in the future of wound care. We present the following article in accordance with the ARRIVE reporting checklist (available at https://dx.doi. org/10.21037/atm-21-2739).

\section{Methods}

\section{Materials}

Taurine was supplied by Sigma-Aldrich (St. Louis, MO, USA). Dulbecco's modified Eagle's medium (DMEM), phosphate-buffered saline (PBS), penicillin, streptomycin, and fetal bovine serum (FBS) were obtained from Thermo Scientific (Danvers, MA, USA). Bovine serum albumin (BSA), 4',6-diamidino-2-phenylindole (DAPI), cell counting kit-8 (CCK8) reagent, and a hematoxylin and eosin (HE) staining kit were purchased from Beyotime Biotechnology (Shanghai, China). Masson's trichrome staining kit was provided by Solarbio Science \& Technology Co., Ltd. (Beijing, China).

\section{Collagen sponge extraction}

The collagen sponge was carefully extracted from the tails of Sprague-Dawley rats. The rats (6 weeks old) were kindly supplied by the Laboratory Animal Center of Wenzhou Medical University. We collected enough rat tails and stored them in $-20{ }^{\circ} \mathrm{C}$ before we started the extraction. 
Briefly, rat tails were thoroughly rinsed and sterilized in $75 \%$ ethanol, which was followed by careful extraction of the tendons within the rat tails. The tendons were cut into small sections, and all the tendons were placed in a $4{ }^{\circ} \mathrm{C}$ environment for 48 hours. Then, the tendons were dissolved in $0.1 \mathrm{M}$ of acetic acid for 5 days until they became sufficiently dry. Next, the $\mathrm{pH}$ of the tendon suspension was adjusted to 7.0 and the suspension was centrifuged at $13,000 \mathrm{~g}$ for 10 minutes. After centrifugation, the pellet was evenly distributed in a $35-\mathrm{mm}$ petri dish. Finally, the pellet was frozen in $-80^{\circ} \mathrm{C}$ and lyophilized to obtain the collagen sponge. The collagen sponge was punched into round discs (7 $\mathrm{mm}$ in diameter) and disinfected with $24-\mathrm{h}$ ultraviolet radiation before further application.

\section{Tau@Col preparation and characterization}

For the loading of Tau to the collagen sponge, the freezedried collagen sponge was incubated in $10 \mu \mathrm{L}$ of Tau solution $(0.075 \mathrm{mg} / \mu \mathrm{L}$ in $0.9 \%$ saline) for 30 minutes. The total amount of taurine in the Tau@Col was $0.75 \mathrm{mg}$. Then, the sponge was freeze-dried to obtain Tau@Col. The loading efficiency of Tau in the collagen sponge was indirectly quantified by measuring the unencapsulated Tau via highperformance liquid chromatography (HPLC, Agilent 1100, USA). The unencapsulated Tau sample was collected by mixing the supernatant of the Tau solution after collagen sponge incubation and the washing solution of the collagen sponge after Tau encapsulation. The Tau sample was pretreated with o-phthalaldehyde for 2 minutes, and then immediately loaded onto the HPLC system for quantification [C18 reversed phase column; acetonitrile: sodium acetate buffer $(\mathrm{pH} 5.3)=40: 60$ at $330 \mathrm{~nm}$; flow rate $=1.0 \mathrm{~mL} / \mathrm{min}]$. In addition, the microstructure of Tau@Col was observed by a scanning electron microscope (SEM, VEGA3, TESCAN, Brno, Czech Republic).

\section{In vitro Tau release behavior}

For the release study, Tau@Col was immersed in 0.9\% saline $(1 \mathrm{~mL}, \mathrm{pH} 7.4)$ at $37{ }^{\circ} \mathrm{C}$ in a centrifuge tube. At predetermined time points $(0.5,1,2,3,4,5,6,7,8$, 9, and 10 days), the released solution was collected, and $1 \mathrm{~mL}$ of fresh $0.9 \%$ saline was refilled into the centrifuge tube. The release samples were stored at $-80{ }^{\circ} \mathrm{C}$ until all samples had been collected. Finally, the samples were melted, pretreated, and loaded onto the HPLC system (Darmstadt, Germany) for Tau quantification.

\section{Cell culture and viability}

NIH 3 T3 fibroblast cells were cultured in a T75 flask with complete medium (DMEM containing 10\% FBS and 1\% streptomycin-penicillin solution). The culture medium was changed every day. When fibroblasts reached confluence of $90 \%$, they underwent trypsinization for passaging. Cells of passage 8-12 were used for subsequent proliferation experiments. The cytotoxicity of the free taurine on fibroblasts was assessed using the CCK- 8 assay. Briefly, cells were seeded onto a 96-well cell culture plate for 24 hours. Afterwards, different concentrations of taurine were added into the wells and incubated with cells for 24 hours and 48 hours. At last, the cells were mixed with $10 \mu \mathrm{L}$ of CCK8 kit and incubated at $37^{\circ} \mathrm{C}$ for 2 hours. The optical density (OD) of each well was measured at wavelength of $450 \mathrm{~nm}$ using a microplate reader (Molecular Devices, SoftMax R Pro 5, San Jose, CA, USA) to calculate the cell viability.

\section{Tau@Col biocompatibility}

For the evaluation of biocompatibility, NIH 3 T3 cell suspension in culture medium was incubated either with Col or Tau@Col for up to 4 days. Afterwards, Col and Tau@ Col were fixed with paraformaldehyde and dehydrated, and the cell adhesion in Col and Tau@Col were observed by scanning electron microscopy (SEM).

\section{In vivo wound healing}

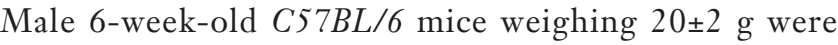
obtained from the Shanghai Slake Laboratory Animal Center (Shanghai, China). The mice were housed in pathogen-free environments with a 12-hour light/dark cycle at $24 \pm 2{ }^{\circ} \mathrm{C}$, and received food and water ad libitum. They were housed in isolation to prevent fighting and aggravation to the wounds. All the animals were quarantined for 1 week before treatment. All manipulations were performed using aseptic techniques, and all animal procedures were performed following the protocol approved by the guidelines of the Animal Ethics Committee of Wenzhou Medical University. The International Code of Ethics and the National Institutes of Health guidelines for the care and use of laboratory animals were also strictly followed. All mice were treated humanely throughout the experimental period and were anesthetized with $4 \%$ chloral hydrate $(0.4 \mathrm{mg} / \mathrm{kg}$ body weight). The dorsal hair was removed, and the surgical areas were sterilized with sterile cotton 
soaked with $70 \%$ ethanol. For each mouse, two silicone rings (internal diameter: $8 \mathrm{~mm}$; thickness: $0.5 \mathrm{~mm}$ ) were symmetrically stitched onto the dorsal skin, where 2 fullthickness excisional wounds were then established with a 6-mm biopsy punch. Twenty-four mice were randomly divided into four groups $(\mathrm{n}=6)$ : group I was applied with $10 \mu \mathrm{L} 0.9 \%$ saline (control); group II was treated with free Tau $(0.75 \mathrm{mg}$ Tau in $10 \mu \mathrm{L} 0.9 \%$ saline); group III received the collagen sponge without encapsulated Tau (Col); group IV was treated with Tau@Col (equivalent to $0.75 \mathrm{mg} \mathrm{Tau}$ ). After the treatments, all wounds were covered with $3 \mathrm{M}$ Tegaderm film sterile bandages to avoid interference with each other. On day 0 (the day wounds received first treatments), $7,10,14$, and 17 , each wound was photographed, based on which the wound size was then measured using Image-Pro (Media Cybernetics, Rockville, MD, USA) for analyzing wound closure rate. The wound closure percentages of different groups were then calculated based the following equation: wound closure $\%=\left(\mathrm{A}_{0}-\right.$ $\left.A_{n}\right) / A_{0} \times 100 \%$, where $A_{0}$ and $A_{n}$ indicate the wound area on day 0 and day n, respectively. At predetermined time point, the mice were sacrificed, and full-thickness wound samples with $1-\mathrm{cm}$ diameter (including the wound area and marginal healthy skin) were harvested and fixed in buffered formaldehyde for histological evaluation.

\section{Histopathologic examination}

For histopathologic analysis, the fixed skin tissue samples were embedded in paraffin, sectioned ( $5 \mu \mathrm{m}$ in thickness), and stained with hematoxylin and eosin (for granulation tissue analysis) or Masson's trichrome staining kit (for collagen deposition analysis). Stained samples were examined by a Nikon light microscope (80i, Tokyo, Japan).

\section{Immunobistochemical staining}

For immunohistochemical analysis, paraffin sections were deparaffinized at $56^{\circ} \mathrm{C}$ by xylene, and then incubated in absolute and $96 \%$ ethanol. The streptavidin-biotin method was used for immunohistochemical detection of cytokeratin expressions. All sections were immersed in $0.01 \mathrm{M}$ sodium citrate buffer ( $\mathrm{pH}$ 6.0) and microwave-treated for a first 10 minutes at $360 \mathrm{~W}$ and a final 5 minutes at $600 \mathrm{~W}$. After rinsed with Tris-buffered saline (TBS, $\mathrm{pH}$ 8.0), the endogenous peroxidase activity of the sections was blocked by a 15 -minute immersion in $1.5 \%$ hydrogen peroxide in distilled water. After incubation overnight at $4{ }^{\circ} \mathrm{C}$ with rabbit polyclonal antibody to cytokeratin antibody (ab9377, for analyzing epidermis regeneration; Abcam, Cambridge, $\mathrm{UK})$ at a 1:300 dilution in TBS, a goat anti-rabbit $(\mathrm{H}+\mathrm{L})$ HRP secondary antibody (Bioworld, Minnesotan, USA) was applied for 20 minutes. Then, diaminobenzidine tetrahydrochloride (DAB) was used as chromogen for visualization of antibody. After staining cell nucleus with hematoxylin, tissue sections were dehydrated and mounted with neutral resin. The stained samples were observed by a Nikon light microscope (80i).

\section{Immunofluorescence staining}

Immunofluorescence staining was performed to detect the expression of various specific markers in the skin after different treatments. The primary antibodies diluted in $1 \%$ BSA used to detecting target proteins were as follows: anti-Ki67 (1:100; ab16667, for analyzing cell proliferation level; Abcam), anti-CD31 (1:20; ab28364, for analyzing vascularization; Abcam), anti-vascular endothelial growth factor (VEGF) (1:200, ab1316; Abcam,, anti-transforming growth factor beta (TGF- $\beta$ ) (1:500; ab215715; Abcam), anti-CD68 (1:100; ab955, for analyzing macrophages; Abcam), and anti-CD163 (1:500; ab182422, for analyzing M2 macrophages, Abcam). The secondary antibodies used included donkey anti-mouse IgG Alexa Flour 488 (1:200; ab150073; Abcam) and donkey anti-rabbit Alexa Fluor 647 (1:200; ab150111; Abcam). The paraffin sections were deparaffinized with xylene, rehydrated with gradient ethanol, operated on with antigen retrieval, blocked with $5 \% \mathrm{BSA}\left(37^{\circ} \mathrm{C}, 30 \mathrm{~min}\right)$, and incubated with the specific primary antibody overnight at $4{ }^{\circ} \mathrm{C}$. After proper rinsing with PBS, the sections were incubated with corresponding secondary fluorescent antibodies and counterstained with DAPI in dark. All fluorescent images were captured using a Nikon confocal microscope (A1 PLUS) and further analyzed by using the Nis-element advanced research software (Nikon) followed by subsequent statistical analysis.

\section{Statistical analysis}

The data were collected and analyzed, and are reported as mean \pm standard deviation (SD). For each analysis, all statistical differences were performed using one-way analysis of variance (ANOVA) followed by Tukey's test with GraphPad Prism 6 software (GraphPad Software Inc., San Diego, CA, USA). For all analysis, a significant difference was indicated as follows: ${ }^{*} \mathrm{P}<0.05,{ }^{* *} \mathrm{P}<0.01$, ${ }^{* * *} \mathrm{P}<0.001$. In 

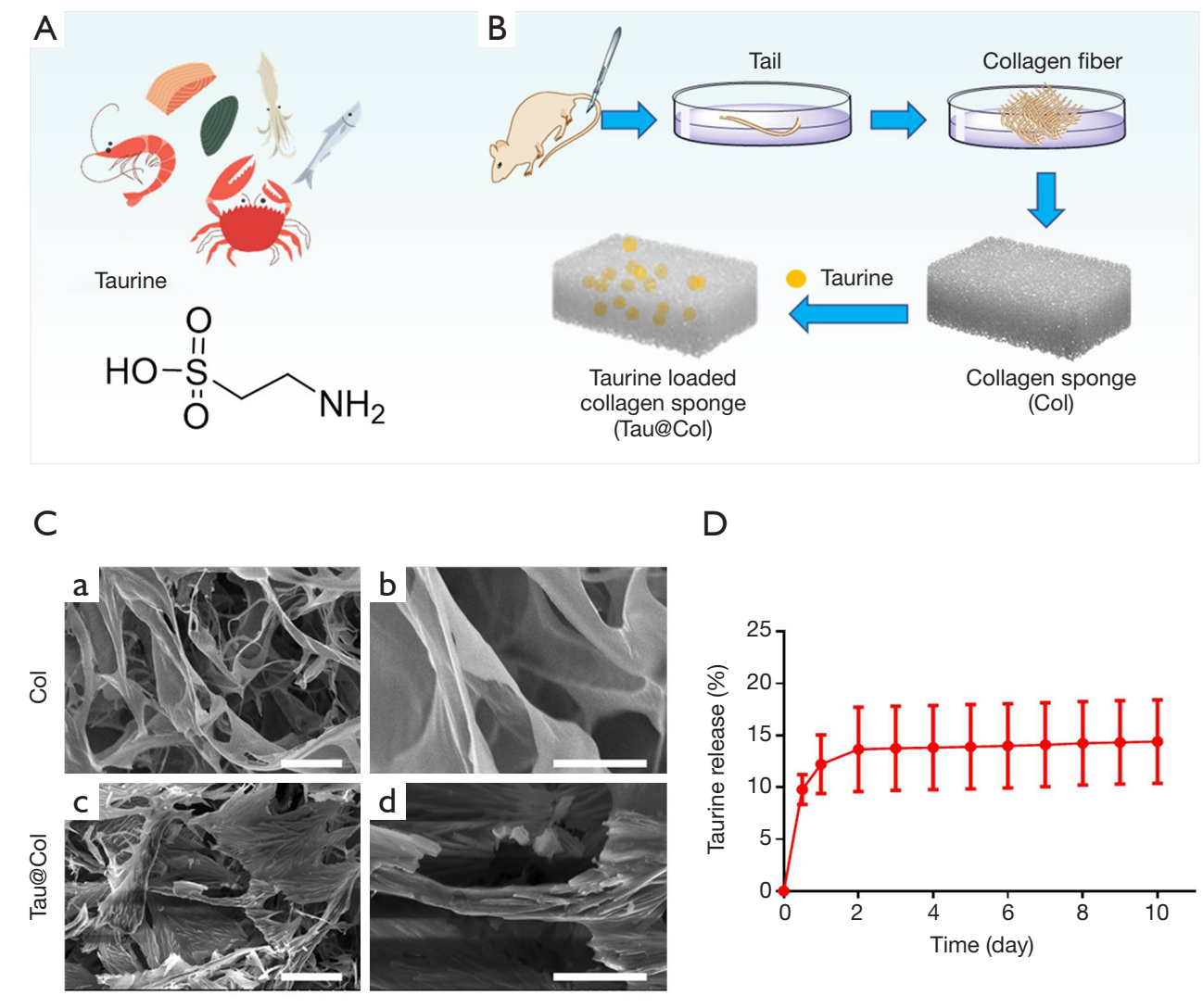

D

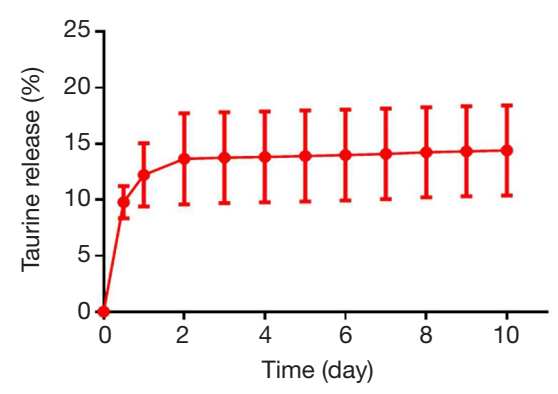

Figure 1 Preparation and characterization of a collagen sponge (Col) and a taurine-loaded collagen sponge (Tau@Col). (A) Origin and chemical structure of taurine (Tau). (B) Schematic illustration of the preparation of Col and Tau@Col. (C) Representative SEM images of Col (a and b) and Tau@Col (c and d). Scale bars are $50 \mu \mathrm{m}$ (a and c) and $20 \mu \mathrm{m}$ (b and d). (D) Taurine release profile of Tau@Col at $37{ }^{\circ} \mathrm{C}$.

each analysis, the unit (n) indicates the sample amount in each group.

\section{Results and discussion}

\section{Preparation and characterization of Tau@Col}

As one of the most abundant free amino acids in mammals, taurine is also found freely in many foods (Figure 1A). Here in this study, in order to provide dermal wounds with a supportive scaffold for tissue regeneration, Tau was loaded into a rat tail-derived collagen sponge to form a novel collagen dressing for Tau delivery (Tau@Col; Figure 1B). SEM results showed that both Col and Tau@Col exhibited microporous and interconnected interior structures with ordered internal arrangement (Figure 1C). Then, the taurine-releasing profile from the Tau@Col scaffold was investigated by incubating Tau@Col in $0.9 \%$ saline for 10 days. As presented in Figure 1D, it was found that taurine could be released from the sponge composite for more than 10 days, with a cumulative release amount of taurine around $14.40 \% \pm 8.04 \%$ as indicated by the data curve, demonstrating the successful loading and sustained release of taurine in the collagen sponge. Overall, these data prove that the Tau-encapsulated collagen dressing was successfully fabricated, and the dressing exhibited effectiveness in controlling the release of Tau, which would be beneficial for the long-term application of Tau in the wound bed.

\section{Cyto-biocompatibility of Tau@Col}

To determine the cyto-compatibility of the taurine, we evaluated the in vitro viability of NIH $3 \mathrm{~T} 3$ mouse embryonic fibroblast cells upon interaction with taurine and Tau@Col. Fibroblasts are one of the predominent cell types of the skin, playing an essential role throughout the wound repair process (26). As indicated in Figure $2 A, B$, negligible cytotoxicity was detected when the cells were treated with Tau at concentrations ranging from 10 to $100 \mathrm{mM}$, proving 

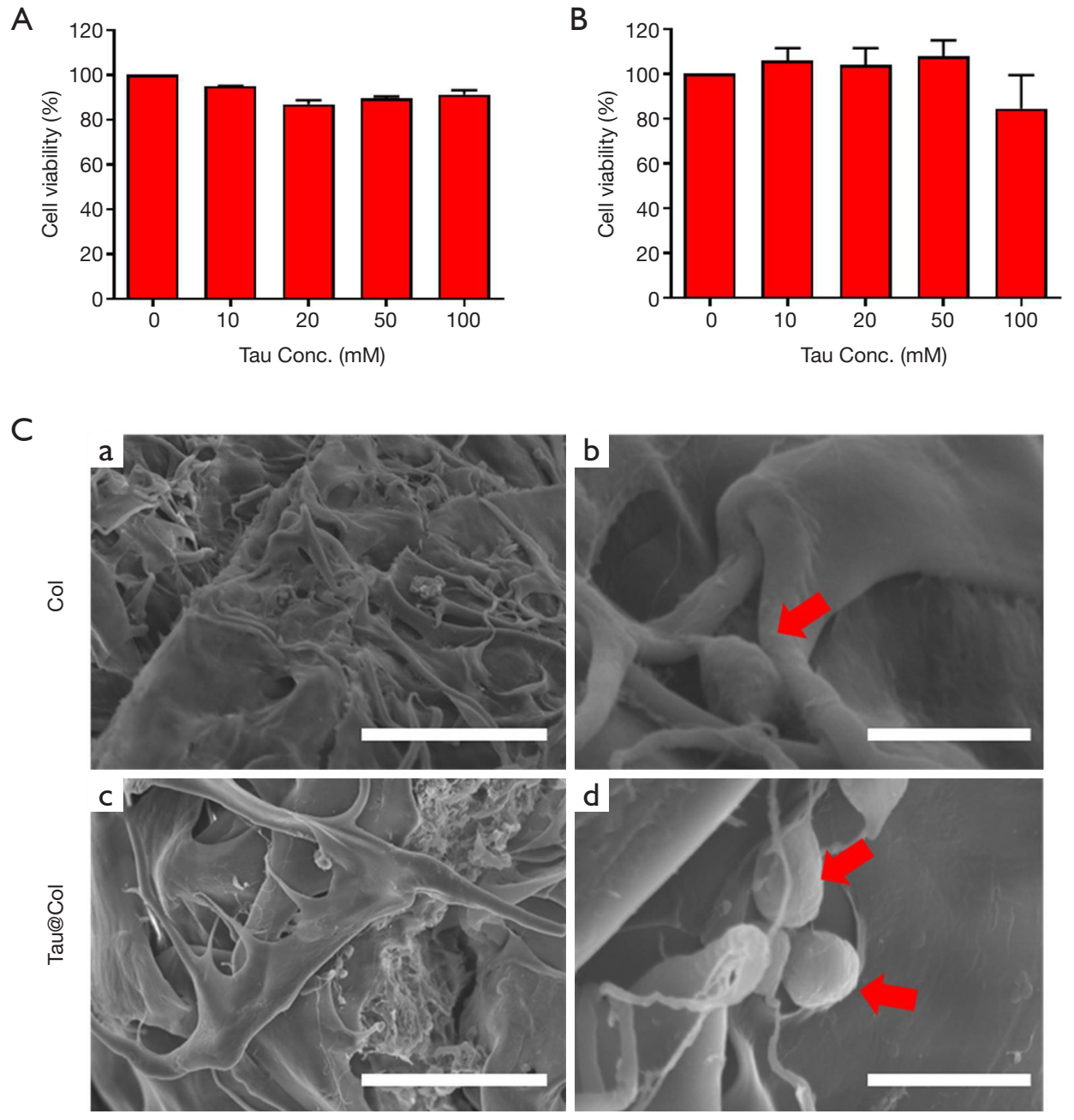

Figure 2 Biocompatibility of Tau@Col on fibroblasts. (A) Cell viability of coincubation of cells with different concentrations of Tau for 24 hours ( $n=6$ ). (B) Cell viability of coincubation of cells with different concentrations of Tau for 48 hours (n=6). (C) Representative SEM images after 4 days of incubation cells with the Col and Tau@Col. The red arrow (b and d) indicates the cells. Scale bar =100 $\mu m(a$ and $c)$ and $10 \mu \mathrm{m}$ (b and d).

the high biocompatibility of Tau. After the loading of Tau into the collagen scaffold (Tau@Col), the biocompatibility of Tau@Col was further tested. Briefly, fibroblasts (NIH 3T3) were co-incubated with Tau@Col for 4 days, and SEM was used to investigate the cell morphology at the surface and central areas of vertical cross-sections of this scaffold to assess the cell situation. The collagen sponge was used as control. As shown in Figure 2C, SEM images showed that the cells could successfully infiltrate into both Col and Tau@Col, and both exhibited round and normal cell morphology, revealing the excellent biocompatibility of $\mathrm{Col}$ and the Tau@Col sponge. This in vitro study of collagen and Tau@Col suggested the safety of application of Tau@
Col in vivo.

\section{Tau@Col accelerated wound closure in vivo}

As the cyto-compatibility of Tau and Tau@Col were confirmed, the biological activity of Tau@Col in the in vivo acute full-thickness excision rat model was further evaluated by comparing it with the free taurine and collagen sponge without taurine encapsulation (Col). Consequently, 6 mice were involved in the experiments from day 0 to 7 , and 3 mice were involved from day 8 to 17 . Figure $3 A$ shows the representative sequential photographs of wounds in 4 groups on day $0,7,10,14$, and 17 , respectively. The wounds 

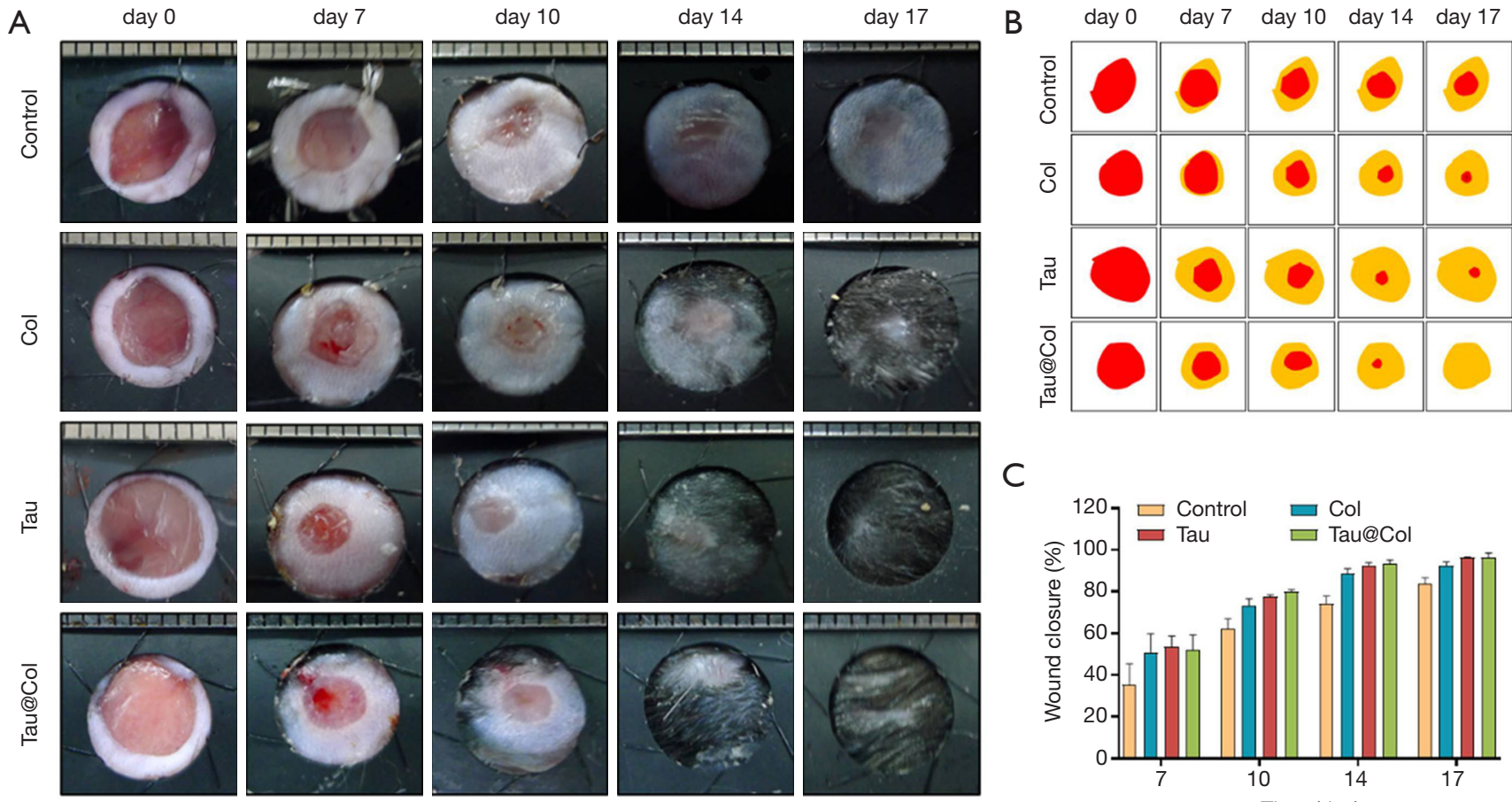

C

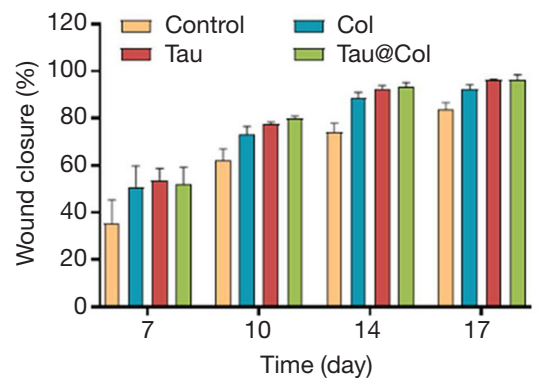

Figure 3 Tau@Col administration in mouse dermal wound models. (A) Representative photographs of the wounds upon different treatments (ruler unit $=1 \mathrm{~mm}$ ). (B) Schematic diagram of the in vivo wound resurface traces of 4 groups over the 17-day course. Orange color represents the initial wound areas, while the red color represents the wound areas on a specific day. (C) The wound closure rate in mice receiving different treatments. Each point represents the mean \pm SD of 4 wounds.

treated with taurine and Tau@Col indeed accelerated the wound closure at day 7 and day 14, but the Tau@Coltreated group exhibited faster wound closure with better skin appearance than other groups. Particularly, after 17 days, the Tau@Col-treated wounds were almost healed with scarless skin, while the collagen sponge and taurine groups still retained large residual wounds. This pattern was much more obvious in the wound tracing results that are displayed in Figure 3B. Quantitatively, Figure 3C statistically summarizes the wound closure rate for each group, which was consistent with the above visual inspection of wound areas. The healing rate of the Tau@Col-treated group showed the fastest wound closure rate with the highest wound closure rate of about $99.6 \%$ at day 17 , followed by the taurine $(\sim 89.9 \%)$ and collagen sponge $(80.2 \%)$ treatments. The control $0.9 \%$ saline group showed the lowest healing rate of $79.3 \%$ with the wound still covered with eschar on day 17. The Tau@Col group showed a comparable and much higher healing rate than did the free taurine and collagen group, indicating the sustained release of taurine from the collagen sponge has a positive effect on the wound repair process.

\section{Tau@Col enhanced granulation formation, along with collagen deposition and re-epithelialization}

The wound healing efficacy of Tau@Col was further evaluated at the tissue level by histological staining of the wound tissue section. Granulation tissue appears as the newly-formed construction filling in the skin defect, which mainly consists of connective tissues as well as capillaries. Granulation tissue formation usually begins to appear at the wound site after 2 days and is considered as an especially important step for wound regeneration. Thus, the granulation tissue formation of the regenerated wound skin on day 7 and day 17 were detected by hematoxylin and eosin (HE) staining (Figure 4A) and Masson's trichrome staining (Figure $4 B$ ). For each histological analysis on each time point, 3 mice were involved. In Figure 4A, the control wound remained at an unhealed dermal gap of $4.4 \pm 0.03 \mathrm{~mm}$ on day 7, while the Tau@Col treatment effectively accelerated granulation tissue formation from both wound 
A

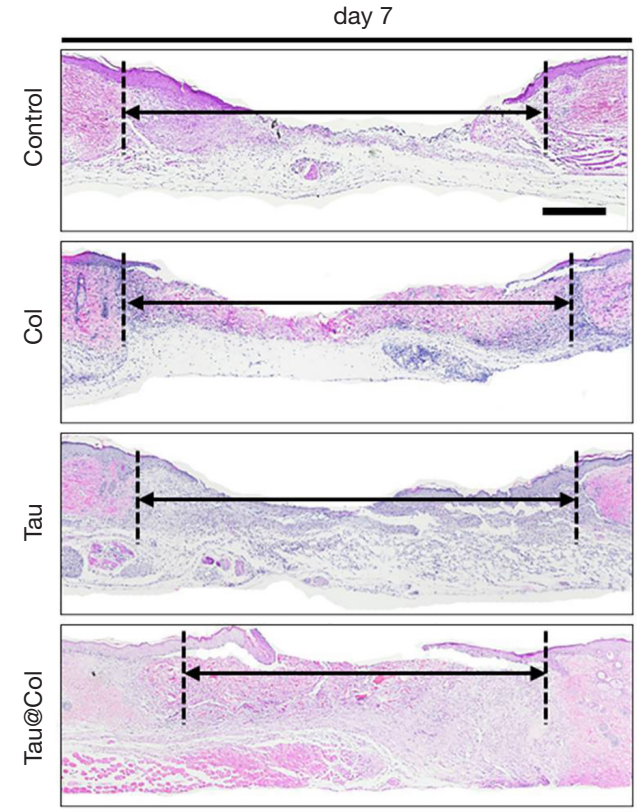

B

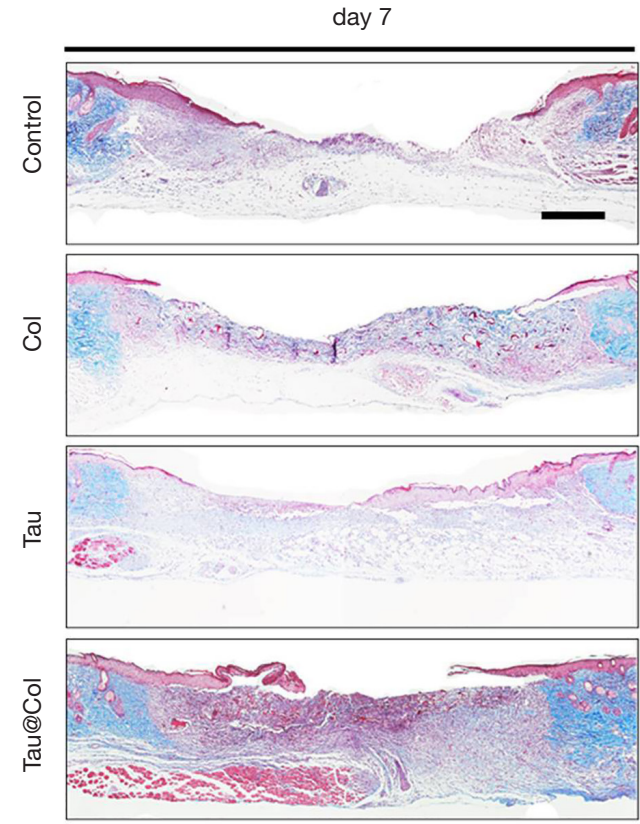

day 17
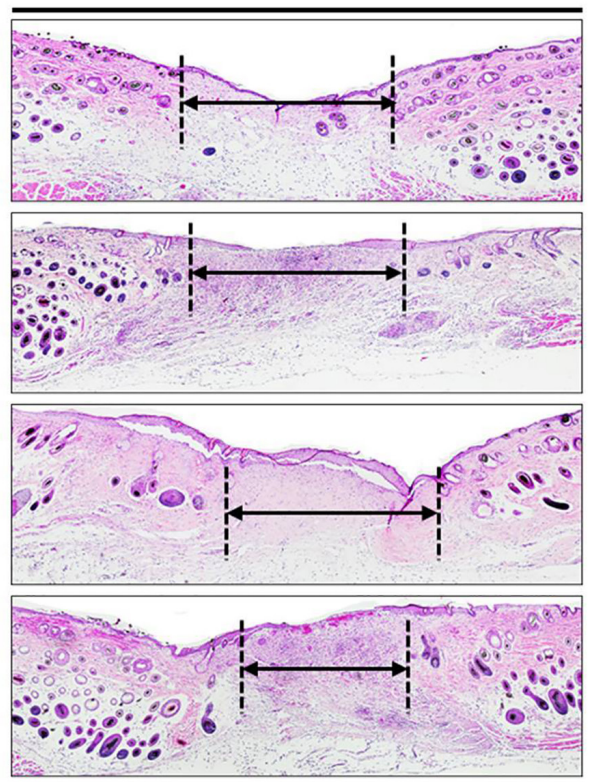

day 17

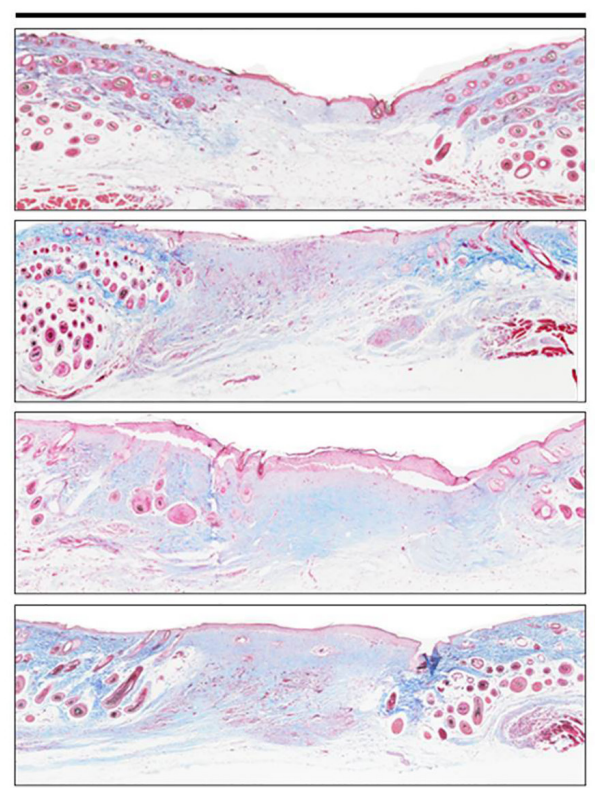

Figure 4 Tau@Col promoted granulation formation and collagen deposition in wound sites. (A) HE staining of control-, Col-, Tau-, and Tau@Col-administered wound sections on day 7 (left) and 17 (right). Double-headed arrows indicate the epidermal gap. (B) Masson's trichrome staining indicating collagen deposition in the wound center upon different treatments at day 7 (left) and 17 (right). Scale bar $=1 \mathrm{~mm}$.

edges, leaving a narrow dermal gap of only $3.5 \pm 0.02 \mathrm{~mm}$ $(\mathrm{P}<0.001)$, suggesting a significant positive effect of Tau@ Col upon the wound site in promoting wound closure. The other 2 groups also showed significant reductions of $4.5 \pm 0.02 \mathrm{~mm}$ for the Col treatment and $4.6 \pm 0.01 \mathrm{~mm}$ for the free Tau treatment. After another 10 days, all groups exhibited larger areas of the healed gap. Specifically, compared to control group $(2.2 \pm 0.07 \mathrm{~mm})$, the Tau@Col group showed significantly enhanced granulation formation, with $1.7 \pm 0.02 \mathrm{~mm}$ of unhealed tissue. 
A
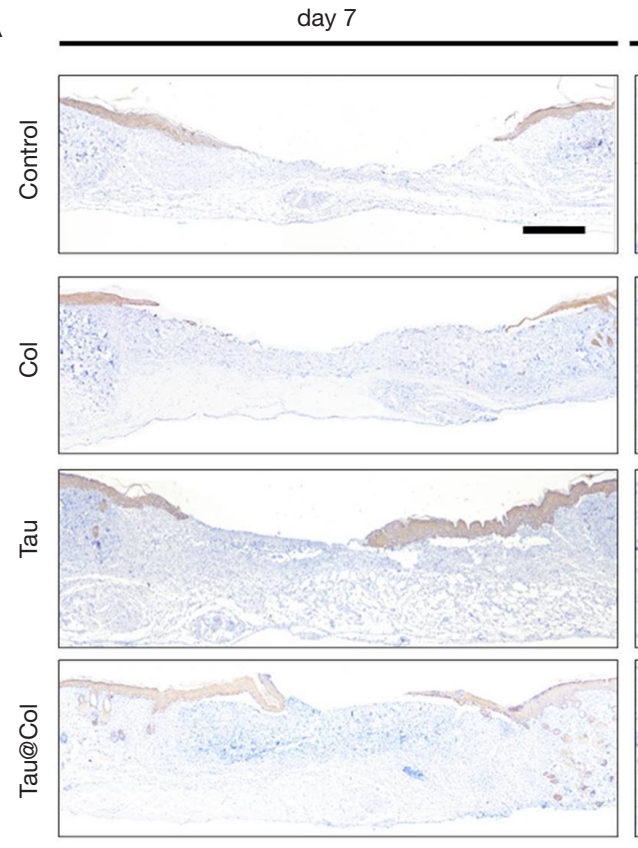

B

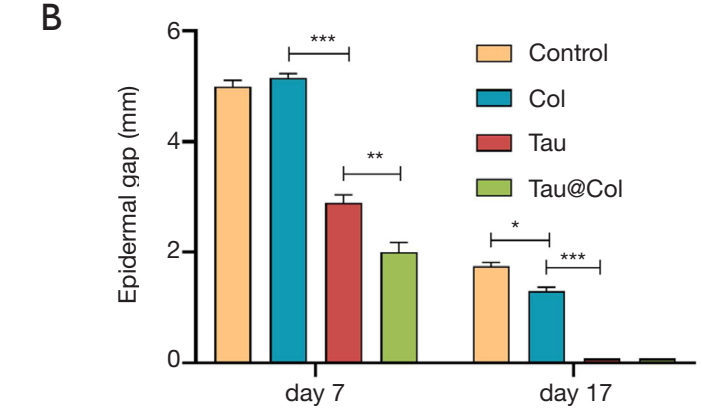

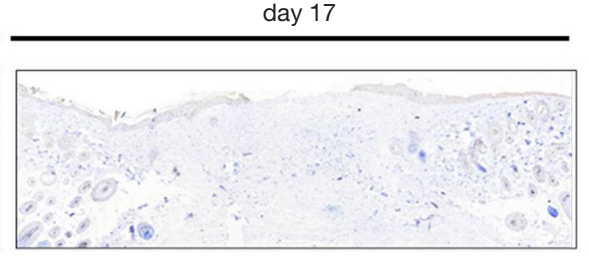
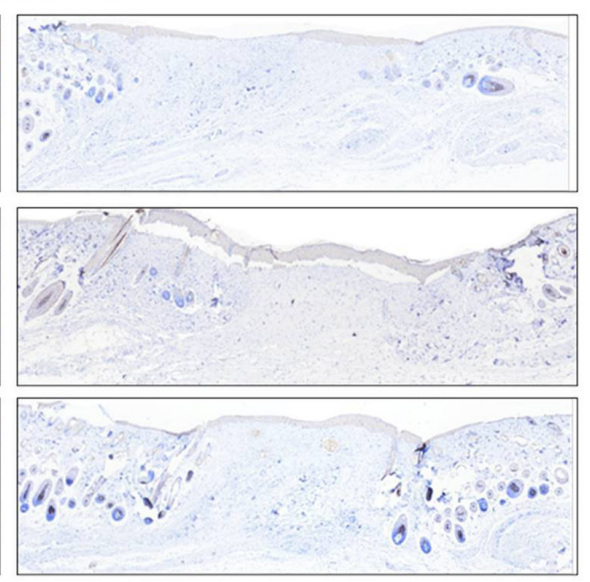

Figure 5 Enhanced epidermis regeneration after Tau@Col application. (A) Representative images of immunohistochemistry staining of cytokeratin within wound sections on day 7 (left) and 17 (right) post wounding. Double-headed arrows indicate an epidermal gap. Scale bar: $1 \mathrm{~mm}$. (B) Quantification of the epidermal gap upon the wound site after treatment of the 4 groups. All data are expressed as mean \pm $\mathrm{SD}$ and were analyzed using one-way analysis of variance (ANOVA). Significant differences are indicated as follows: ${ }^{*} \mathrm{P}<0.05$, ${ }^{* *} \mathrm{P}<0.01$, and ${ }^{* * *} \mathrm{P}<0.001 ; \mathrm{n}>3$.

Collagen deposition is another pivotal factor in evaluating the wound repair stage. We performed Masson's trichrome staining, in which collagen fibers and cell nuclei were stained with blue while muscle tissue was stained with red to analyze collagen deposition on day 7 and 17 (Figure 4B). On day 7, when the wounds were treated with Tau@Col, there were considerably denser collagen fibers produced in the wound bed compared with the other three groups. Similarly, on day 17, Tau@Col group had the highest collagen deposition percentage, followed by the $\mathrm{Col}$ and free Tau groups, with the control group showing the lowest collagen deposition. Compared with the other groups, the underlying collagen fibers of the Tau@Col group were well organized and much more like normal skin with newly formed blood vessels and skin appendage on day 17. The enhanced granulation tissue formation along with the improved extensive collagen deposition confirmed the accelerated wound healing property of Tau@Col.

Finally, the re-epithelialization of wounds was evaluated by immunochemistry staining of cytokeratin (epidermisspecific protein), as the re-epithelialization directly reflected the wound closure level. For cytokeratin analysis at each point-in-time, 3 mice were involved. As shown in Figure $5 \mathrm{~A}$, the Tau@Col-treated group exhibited the fastest re- 
epithelialization of all groups on both day 7 and 17, as evidenced by the greater expression of cytokeratin in the epidermal layer. Of note, on day 17, the taurine- and Tau@ Col-treated groups showed a complete epidermal cover above the healed wound. The epithelialization of the wound incisions increased in the order of Tau@Col > Tau > Col > control, demonstrating the positive effect of Tau@Col on epidermal regeneration as a result of the retained taurine activity via its incorporation into the collagen hydrogel. Figure $5 B$ shows the quantitative comparison of the treatment groups: the control had a $5.0 \pm 0.13 \mathrm{~mm}$ unhealed epidermal gap, the Tau@Col group exhibited a significantly accelerated epidermal gap $(2.0 \pm 0.19 \mathrm{~mm})$ on day 7 , while the free taurine group had an epidermal gap of $2.9 \pm 0.16 \mathrm{~mm}$. On day 17, both the taurine and Tau@Col showed no epidermal gap, which was consistent with the fact that a complete integrated cytokeratin had covered the newly regenerated granulation. The observations of epidermal covering were basically in line with the visual wound closure rate shown in Figure 3, which might have resulted from the therapeutic effects of taurine from the Tau@Col. Thus, the overall performance of Tau@Col sponge has validated our hypothesis that the controlled release of taurine with an extracellular matrix (ECM)-mimetic biomaterial is an effective approach to promoting wound regeneration.

\section{Tau@Col promoted cell proliferation}

In proliferation stage of wound healing, dermal fibroblasts are activated to proliferate, migrate and produce collagen for filling in the defect. When angiogenesis occurs, endothelial cells would proliferate for transportation of nutrient and oxygen. Keratinocytes would proliferate and migrate for re-epithelialization (27). Our findings demonstrate that taurine plays an essential role in stimulating cell proliferative activity (28-32). Therefore, in order to better understand the wound healing mechanism of Tau@Col, immunofluorescence staining of Ki67 (a proliferation indicator) in the wound tissue sections was extensively studied to assess the proliferative activity of cells. For Ki67 analysis, 3 mice were involved. As revealed in Figure 6A, the Tau@Col group exhibited the highest level of Ki67-expressing cells in the wound center, followed by the Tau, Col, and control groups. Figure $6 B$ shows that, compared to the control group, the Tau and Col groups positive-cell percentage was relatively higher level. The Tau group and Col group showed a 2.7-fold and 1.6-fold increase over the control group, respectively. The Tau@
Col group exerted the highest level of Ki67-positive cells (5.7-fold), indicating the proliferative effect generated by the prolonged release of taurine from the Tau@Col sponge. These results were in line with the data from HE staining, MTS, and cytokeratin testing of the wound site, indicating that the enhanced healing ability of Tau@Col may be stimulated by larger amount of granulation and collagen matrix secreted due to a greater degree of proliferation.

\section{Tau@Col upregulated the expressions of endogenous TGF- $\beta$ and VEGF}

We further investigated the expression of 2 important cytokines, TGF- $\beta$ and VEGF, in the wound beds by immunofluorescence staining. TGF- $\beta$ is a multipotent growth factor that controls cell proliferation, differentiation, apoptosis, and matrix production $(33,34)$, while VEGF is one of the key factors for regeneration of new blood vessels which supply the necessary oxygen and nutrients in the wound beds $(35,36)$. For each analysis, 3 mice were involved. As indicated in Figure 7A,B, the control presented the lowest expression of TGF- $\beta$. Both Col- and Tau-treated groups significantly enhanced the TGF- $\beta$ expression by 3.2-fold and 3.5-fold that of the control group, respectively. Furthermore, the Tau@Col-treated group had the highest TGF- $\beta$-positive expression at almost 7.3 -fold that of the control. Consistent with the TGF- $\beta$ findings, the control, Col, Tau, and Tau@Col groups showed the lowest to highest expressions of VEGF, respectively (Figure 7C,D). The enhanced expressions of VEGF and TGF- $\beta$ in the wound beds further confirmed the positive effect of Tau@Col in promoting wound healing, which indeed stimulated cell proliferation, collagen deposition, and neovascularization.

\section{Tau@Col promoted macrophage polarization to inbibit inflammation}

Macrophage infiltration plays a vital role in wound regeneration, which usually includes M1 macrophage (the proinflammatory phenotype) or M2 macrophage (the anti-inflammatory phenotype). In the initial, the inflammation occurs for protecting skin tissue from invasion of pathogens. However, the inflammation phase needs to transit to the proliferation phase for subsequent tissue regeneration. It has been reported that tuning the macrophage polarization from M1 to M2 would effectively enhance wound repair (37). As inflammation is closely related to oxidative stress, the 2 subtypes of macrophage 


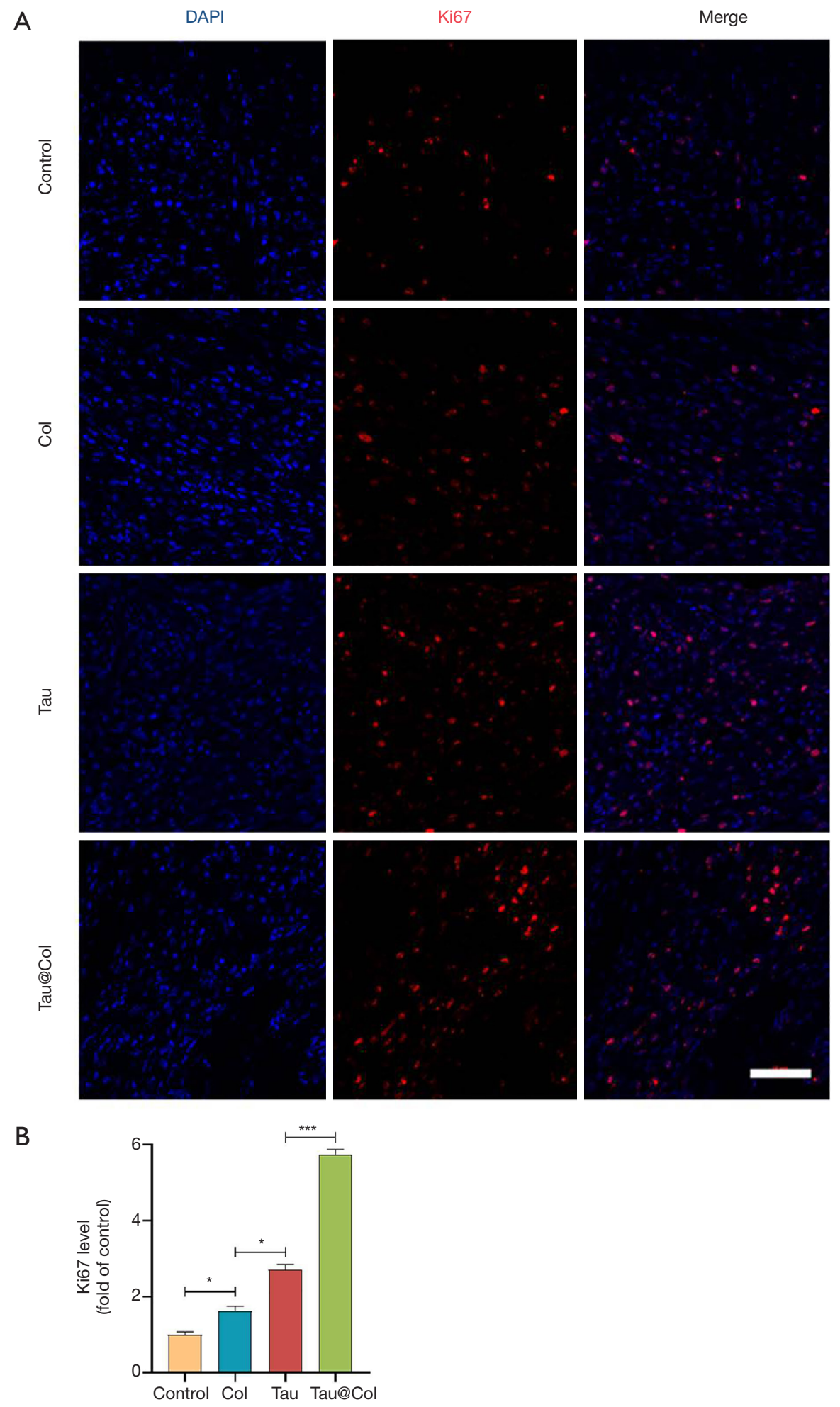

Figure 6 Tau@Col up-regulated cellular proliferation level within wound tissue. (A) Immunofluorescence staining of cell proliferation indicator Ki67 in wound beds on day 7. Scale bar $=50 \mu \mathrm{m}$. (B) Quantification of the Ki67 in the 4 groups. All data are expressed as mean $\pm \mathrm{SD}$ and were analyzed using one-way analysis of variance (ANOVA). Significant differences are indicated as follows: ${ }^{*} \mathrm{P}<0.05$, and ${ }^{* * *} \mathrm{P}<0.001 ; \mathrm{n}>3$. 
A

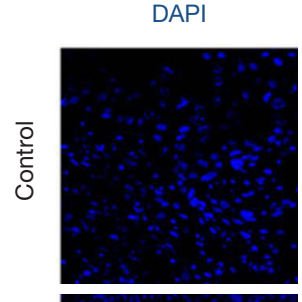

$\bar{\circlearrowright}$

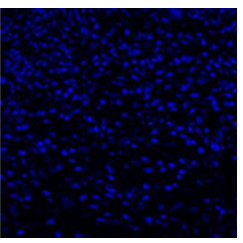

డై
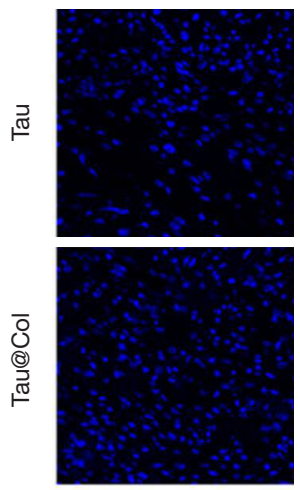

B
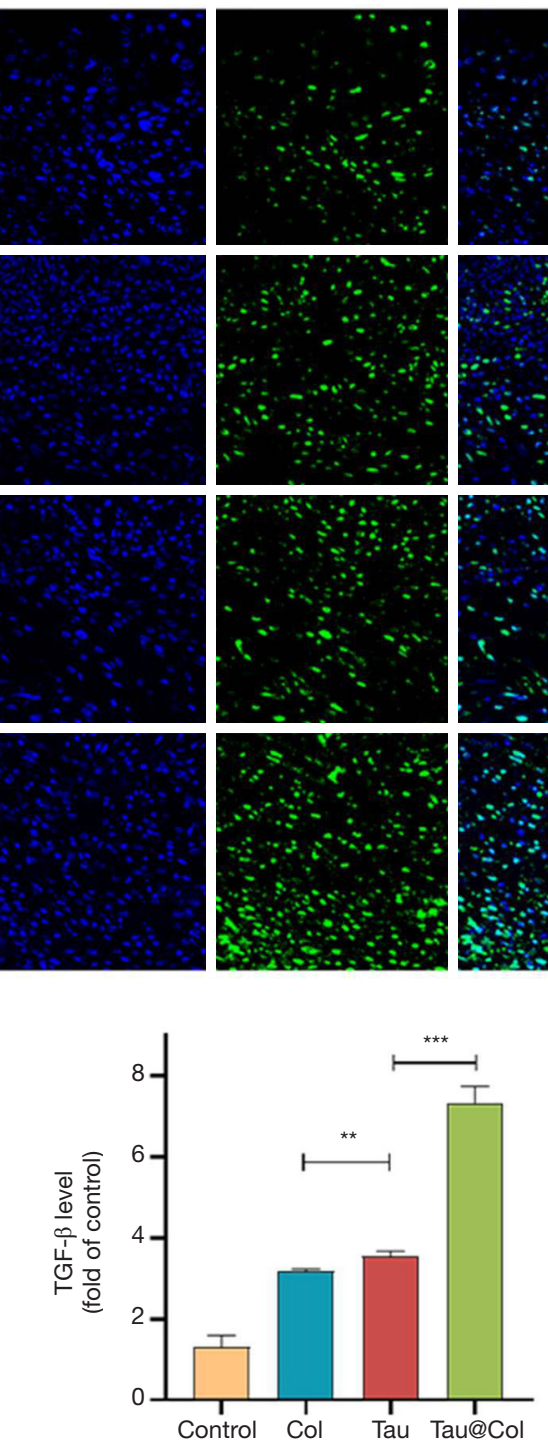

C
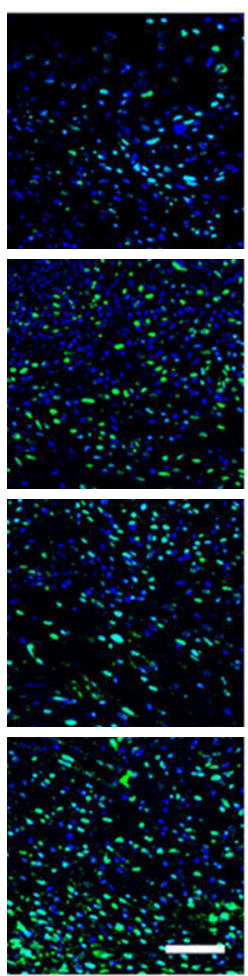

D

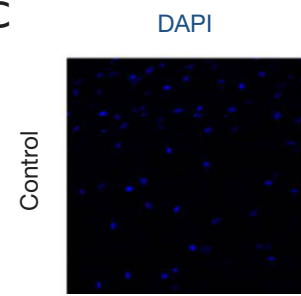

VEGF
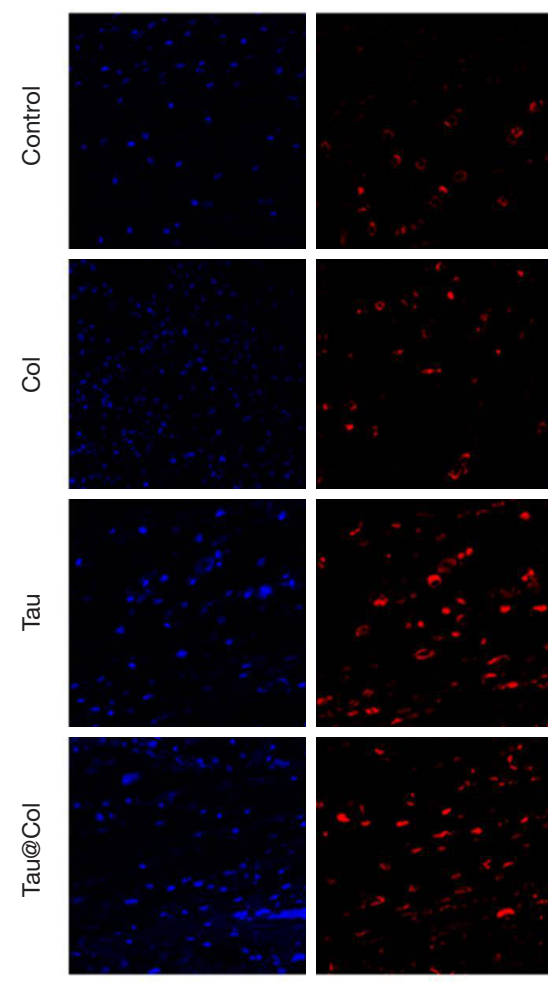
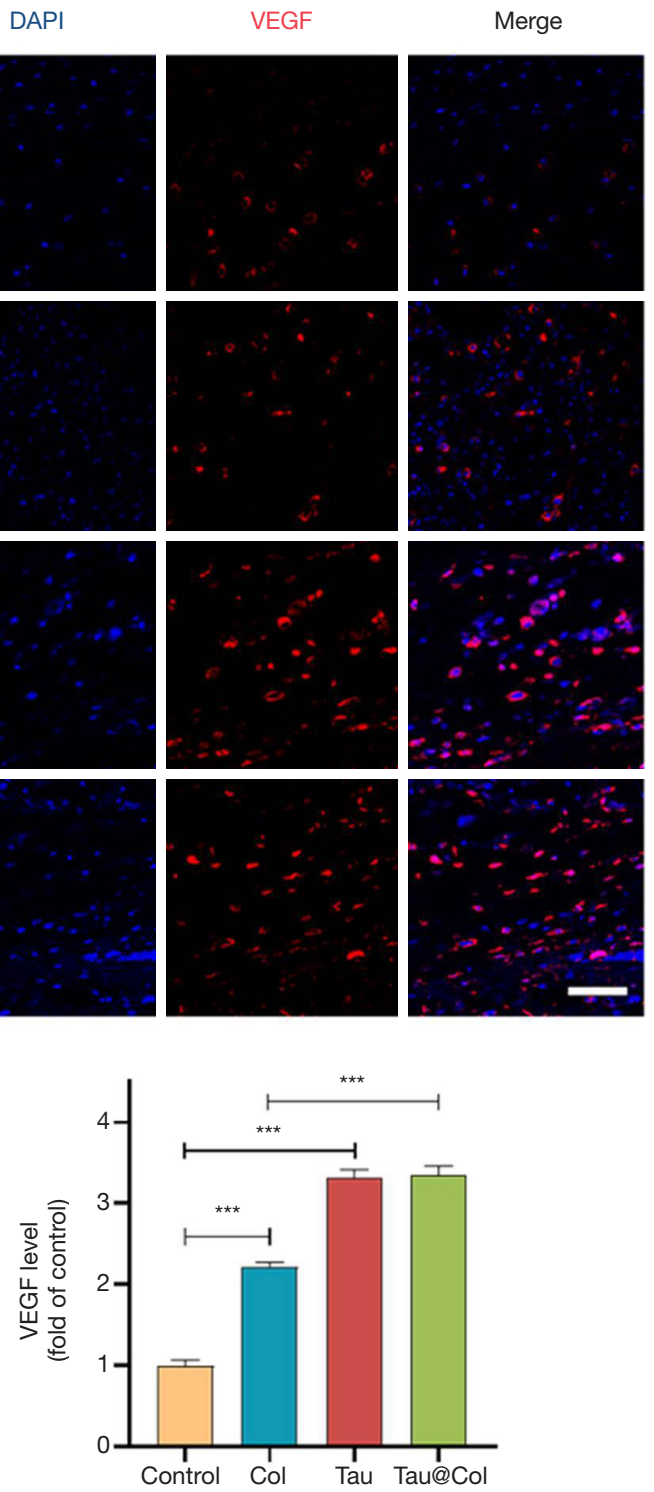

Figure 7 Tau@Col induced elevated expression level of TGF- $\beta$ and VEGF proteins. (A) Immunofluorescence staining of cytokine TGF- $\beta$. Scale bar $=50 \mu \mathrm{m}$. (B) Quantification of TGF- $\beta$-positive cells on day 7 in the 4 groups. (C) Immunofluorescence staining of VEGF. Scale bar $=50 \mu \mathrm{m}$. (D) Quantification of the VEGF-positive cells on day 7 in the 4 groups. All data are expressed as mean \pm SD and were analyzed using one-way analysis of variance (ANOVA). Significant differences are indicated as follows: ${ }^{* *} \mathrm{P}<0.01$, and ${ }^{* * *} \mathrm{P}<0.001 ; \mathrm{n}>3$.

would reflect the inflammatory response in the wounds. Thus, we evaluated the inflammatory levels through the behavior of macrophage polarization upon the wound site. We chose CD68 as a marker of all the subtypes of macrophages accompanied by CD163 as a label of M2 macrophages, with 3 mice being used for these experiments. As displayed in Figure 8A, the control group exhibited the lowest level of CD163-positive cells. Statistical quantification of CD163 summarized in Figure 8B demonstrates that collagen sponge, free taurine, and Tau@Col groups all showed significantly upregulated M2 macrophage polarization (CD163-positive cells). The percentage of M2 subtype macrophage versus total macrophages was further calculated. As summarize in Figure 8C, the control group had the lowest M2 percentage while the collagen sponge, free taurine, and Tau@Col exhibited higher levels. 

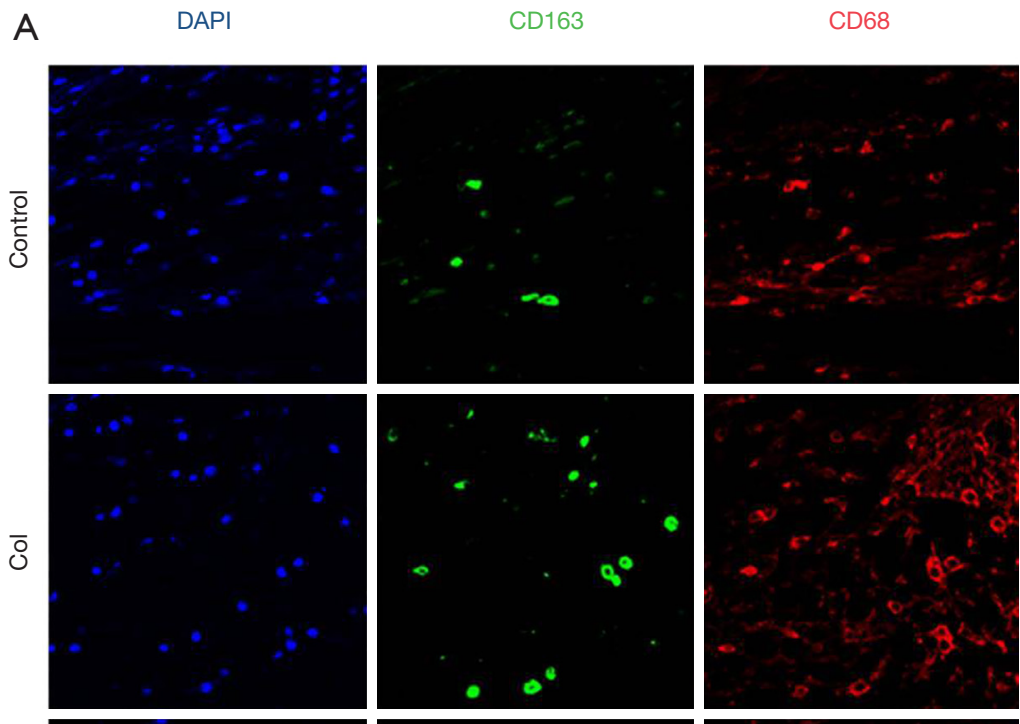

Merge
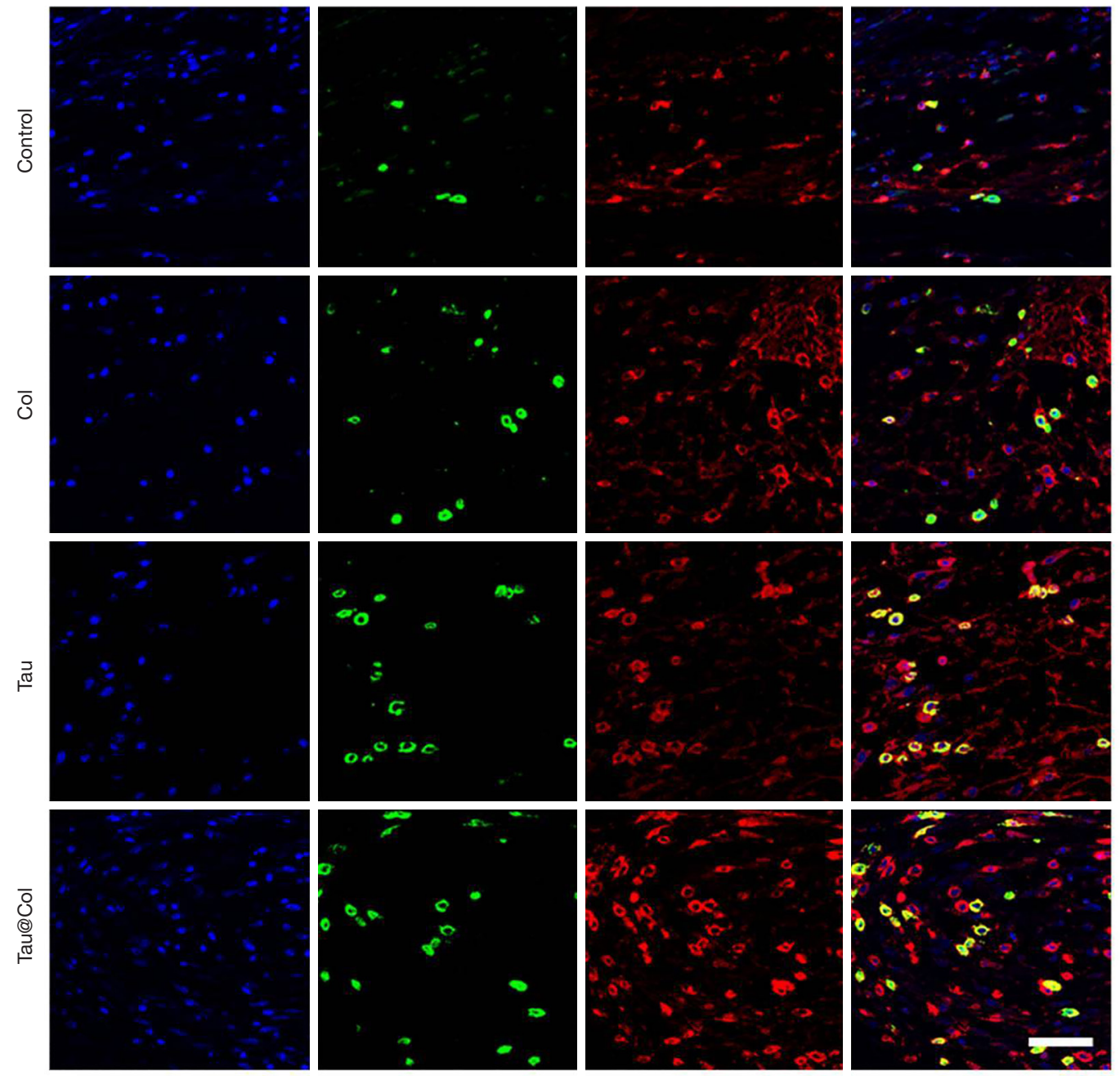

B

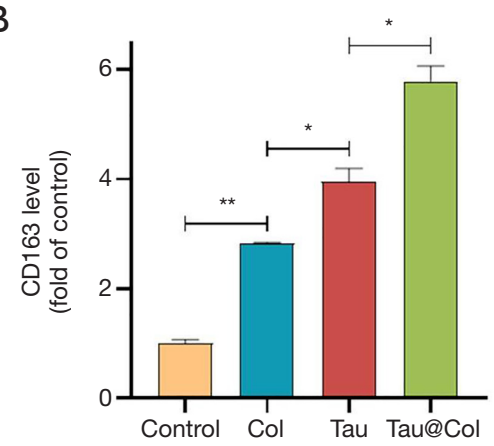

C

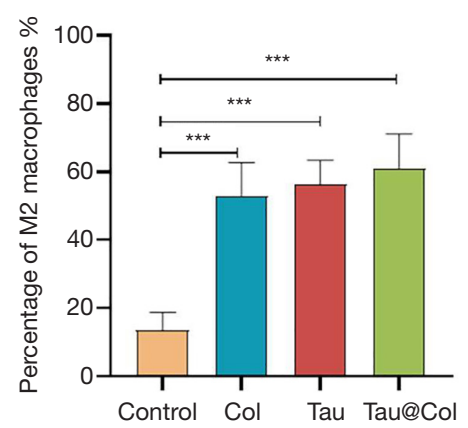

Figure 8 Tau@Col drove significant polarization of wound macrophages towards M2 anti-inflammatory phenotype. (A) Immunofluorescence micrographs of M2 macrophages within the control, free Col-, Tau-, and Tau@Col-treated wounds. Scale bar =50 $\mu$. (B) Quantification of the immunofluorescence staining of CD163-positive cells. (C) Quantification of the percentage of M2 macrophages (CD163/CD68). All data are expressed as mean \pm SD and were analyzed using one-way analysis of variance (ANOVA). Significant differences are indicated as follows: ${ }^{*} \mathrm{P}<0.05,{ }^{* *} \mathrm{P}<0.01$, and ${ }^{* * *} \mathrm{P}<0.001$ as compared to the control group; $\mathrm{n}>5$. 
Most notably, there was a significant increase of M2 polarization levels: compared to the normalized control group $(13.6 \% \pm 4.2 \%)$ : the free Tau group had a 4.1 -fold $(56.2 \% \pm 5.9 \%, \mathrm{P}<0.001)$ increase, while the Tau@Col group had a 4.5 -fold $(61.1 \% \pm 8.1 \%, \mathrm{P}<0.001)$ increase. Therefore, the study of macrophage polarization status confirmed the anti-inflammatory function of the Tau@ Col dressing in accelerating wound healing via driving macrophage polarization towards the anti-inflammatory phenotype.

\section{Conclusions}

Our study evaluated whether loading taurine into a collagen sponge could sustainably release taurine during the wound regeneration process. Our in vitro results showed that taurine had been successfully loaded into the collagen sponge (Col) to form the Tau@Col sponge. The Tau@ Col sponge was further applied in the acute wound model and compared with free taurine (Tau) and collagen (Col) groups. In vivo results revealed that Tau@Col indeed raised wound repair efficiency as evidenced by accelerated wound closure rate, as well as higher levels of granulation formation and collagen deposition. The positive healing effect was further demonstrated through an enhanced cell proliferation rate, upregulated endogenous growth factor expressions, and increased polarization of M2 macrophages. This kind of bioactive taurine-based wound dressing could represent a new strategy in the future clinical application of regenerative materials.

\section{Acknowledgments}

Funding: This work was financially supported by National Natural Science Funding of China (No. 81703105) and Hospital-Level Scientific Research Project of Anqing Municipal Hospital (No. 2021aqykj02).

\section{Footnote}

Reporting Checklist: The authors have completed the ARRIVE reporting checklist. Available at https://dx.doi. org/10.21037/atm-21-2739

Data Sharing Statement: Available at https://dx.doi. org/10.21037/atm-21-2739

Conflicts of Interest: All authors have completed the ICMJE uniform disclosure form (available at https://dx.doi. org/10.21037/atm-21-2739). The authors have no conflicts of interest to declare.

Ethical Statement: The authors are accountable for all aspects of the work in ensuring that questions related to the accuracy or integrity of any part of the work are appropriately investigated and resolved. All animal procedures were performed following the protocol approved by the guidelines of the Animal Ethics Committee of Wenzhou Medical University. The International Code of Ethics and the National Institutes of Health guidelines for the care and use of laboratory animals were also strictly followed.

Open Access Statement: This is an Open Access article distributed in accordance with the Creative Commons Attribution-NonCommercial-NoDerivs 4.0 International License (CC BY-NC-ND 4.0), which permits the noncommercial replication and distribution of the article with the strict proviso that no changes or edits are made and the original work is properly cited (including links to both the formal publication through the relevant DOI and the license). See: https://creativecommons.org/licenses/by-nc-nd/4.0/.

\section{References}

1. Babior BM. Oxygen-dependent microbial killing by phagocytes (first of two parts). $\mathrm{N}$ Engl J Med 1978;298:659-68.

2. Gültekin SE, Sengüven B, Sofuoğlu A, et al. Effect of the Topical Use of the Antioxidant Taurine on the Two Basement Membrane Proteins of Regenerating Oral Gingival Epithelium. J Periodontol 2012;83:127-34.

3. Hameedaldeen A, Liu J, Batres A, et al. FOXO1, TGFbeta regulation and wound healing. Int J Mol Sci 2014;15:16257-69.

4. Wiseman H, Halliwell B. Halliwell. Damage to DNA by reactive oxygen and nitrogen species: role in inflammatory disease and progression to cancer. Biochem J 1996;313:17-29.

5. Cadenas E, Davies KJ. Mitochondrial free radical generation,oxidative stress, and aging. Free Radic Biol Med 2000;29:222-30.

6. White MJ, Heckler FR. Oxygen free radicals and wound healing. Clin Plast Surg 1990;17:473-84.

7. Foschi D, Trabucchi E, Musazzi M, et al. The effects of oxygen free radicals on wound healing. Int J Tissue React 
1988;10:373-79.

8. Li M, Chen J, Shi MT, et al. Electroactive anti-oxidant polyurethane elastomers with shape memory property as non-adherent wound dressing to enhance wound healing. Chemical Engineering Journal 2019;375:121999.

9. Liang Y, Zhao X, Hu T, et al. Mussel-inspired, antibacterial, conductive, antioxidant, injectable composite hydrogel wound dressing to promote the regeneration of infected skin. J Colloid Interface Sci 2019;556:514-28.

10. He J, Liang Y, Shi M, et al. Anti-oxidant electroactive and antibacterial nanofibrous wound dressings based on poly $(\varepsilon-$ caprolactone)/quaternized chitosan-graft-polyaniline for full-thickness skin wound healing. Chemical Engineering Journal 2020;385:123464.

11. Cao X, Wang Y, Wu C, et al. Cathelicidin-OA1, a novel antioxidant peptide identified from an amphibian, accelerates skin wound healing. Sci Rep 2018;8:943.

12. Zhu Y, Cankova Z, Iwanaszko M, et al. Potent laminininspired antioxidant regenerative dressing accelerates wound healing in diabetes. Proc Natl Acad Sci U S A 2018;115:6816-21.

13. Değim Z, Çelebi N, Sayan H, et al. An investigation on skin wound healing in mice with a taurine-chitosan gel formulation. Amino Acids 2002;22:187-98.

14. Sturman JA. Taurine in development. Physiol Rev 1993;73:119-47.

15. Giriş M, Depboylu B, Doğru-Abbasoğlu S, et al. Effect of taurine on oxidative stress and apoptosis-related protein expression in trinitrobenzene sulphonic acidinduced colitis. Clin Exp Immunol 2008;152:102-10.

16. Dinçer S, Babül A, Erdoğan D, et al. Effect of taurine on wound healing. Amino Acids 1996;10:59-71.

17. Schuller-Levis GB, Park E. Taurine and its chloramine: modulators of immunity. Neurochem Res 2004; 29:117-26.

18. Jeon SH, Lee MY, Rahman MM, et al. The antioxidant,taurine reduced lipopolysaccharide (LPS)induced generation of ROS, and activation of MAPKs and Bax in cultured pneumocytes. Pulm Pharmacol Ther 2009;22:562-66.

19. Oliveira MW, Minotto JB, de Oliveira MR, et al. Scavenging and antioxidant potential of physiological taurine concetrations against different reactive oxygen/ nitrogen species. Pharmacol Rep 2010;62:185-93.

20. Marcinkiewicz J, Kontny E. Taurine and inflammatory diseases. Amino Acids 2014;46:7-20.

21. Högström H. Mechanism and prevention of decrease in wound margin strength. Acta Chir Scand Suppl
1987;539:5-63.

22. Ozmeriç N, Ozcan G, Haytaç CM, et al. Chitosan film enriched with an antioxidant agent, taurine, in fenestration defects. J Biomed Mater Res 2000;51:500-03.

23. Sofuoglu A, Sofuoglu IP, Uc D, et al. Evaluation of initial periodontal treatment with taurin gel application in periodontally diseased patients (in Turkish). J Hacettepe Fac Dent 2007;31:9-15.

24. Foos TM, Wu JY. The role of taurine in the central nervous system and the modulation of intracellular calcium homeostasis. Neurochem Res 2002;27:21-6.

25. Zhao HY, Wu J, Zhu JJ, et al. Research Advances in Tissue Engineering Materials for Sustained Release of Growth Factors. Biomed Res Int 2015;2015:808202.

26. M. Takeo, W. Lee, and M. Ito. Wound healing and skin regeneration. Cold Spring Harb Perspect Med 2015;5:a023267.

27. Rodrigues M, Kosaric N, Bonham CA, et al. Wound Healing: A Cellular Perspective. Physiol Rev 2019;99:665-706.

28. Cao R, Bråkenhielm E, Pawliuk R, et al. Angiogenic synergism, vascular stability and improvement of hindlimb ischemia by a combination of PDGF-BB and FGF-2. Nat Med 2003;9 604-13.

29. Ehrbar M, Zeisberger SM, Raeber GP, et al. he role of actively released fibrin-conjugated VEGF for VEGF receptor 2 gene activationand theenhancement ofangiogenesis. Biomaterials 2008;29:1720-9.

30. Nillesen ST, Geutjes PJ, Wismans R, et al. ncreased angiogenesis and blood vessel maturation in acellular collageneheparin scaffolds containing both FGF2 and VEGF. Biomaterials 2007;28:1123-31.

31. Pieper JS, Hafmans T, van Wachem PB, et al. Loading of collageneheparan sulfate matrices with bFGF promotes angiogenesis and tissue generation in rats. J Biomed Mater Res 2002;62:185-94.

32. Richardson TP, Peters MC, Ennett AB, et al. Polymeric system for dual growth factor delivery. Nat Biotechnol 2001;19:1029-34.

33. Ungefroren H, Wellner UF, Keck T, et al. The Small GTPase RAC1B: A Potent Negative Regulator ofand Useful Tool to Study-TGF $\beta$ Signaling. Cancers 2020;12:3475

34. Liu D, Song J, Ji X, et al. PRDM16 Upregulation Induced by MicroRNA-448 Inhibition Alleviates Atherosclerosis via the TGF- $\beta$ Signaling Pathway Inactivation. Front Physiol 2020;11:846.

35. Apte RS, Chen DS, and Ferrara N. VEGF in Signaling 
Page 16 of 16

and Disease: Beyond Discovery and Development. Cell 2019; 176:1248-64.

36. Veith AP, Henderson K, Spencer A, et al. Therapeutic strategies for enhancing angiogenesis in wound healing. Adv Drug Deliv Rev 2019;146:97-125.

Cite this article as: $\mathrm{Wu} \mathrm{L}$, Zhang Q, Li Y, Song W, Chen A, Liu J, Xuan X. Collagen sponge prolongs taurine release for improved wound healing through inflammation inhibition and proliferation stimulation. Ann Transl Med 2021;9(12):1010. doi: 10.21037/atm-21-2739
Wu et al. Sustained taurine-releasing collagen for wound repair

37. Wu J, Chen A, Zhou Y, et al. Novel H2S-Releasing hydrogel for wound repair via in situ polarization of M2 macrophages. Biomaterials 2019;222:119398.

(English Language Editor: J. Gray) 